\title{
Relationship between strain localization and catastrophic rupture
}

\author{
S.W. Hao ${ }^{\text {a,b,e,*, H.Y. Wang }}{ }^{\text {a }}$, M.F. Xia ${ }^{\text {a,c }}$, F.J. Ke ${ }^{\text {a,d }}$, Y.L. Bai ${ }^{\text {a }}$ \\ ${ }^{\text {a }}$ State Key Laboratory of Nonlinear Mechanics, Institute of Mechanics, Chinese Academy of Sciences, Beijing 100080, China \\ ${ }^{\mathrm{b}}$ Graduate University of Chinese Academy of Sciences, Beijing 100039, China \\ ${ }^{\mathrm{c}}$ Department of Physics, Peking University, Beijing 100871, China \\ d Department of Applied Physics, Beihang University, Beijing 100083, China \\ e School of Civil Engineering and Mechanics, Yanshan University, Qinhuangdao 066004, China
}

Available online 22 May 2007

\begin{abstract}
In order to explore a prior warning to catastrophic rupture of heterogeneous media, like rocks, the present study investigates the relationship between surface strain localization and catastrophic rupture. Instrumented observations on the evolution of surface strain field and the catastrophic rupture of a rock under uniaxial compression were carried out. It is found that the evolution of surface strain field displays two phases: at the early stage, the strain field keeps nearly uniform with weak fluctuations increasing slowly; but at the stage prior to catastrophic rupture, a certain accelerating localization develops and a localized zone emerges. Based on the measurements, an analysis was performed with local mean-field approximation. More importantly, it is found that the scale of localized zone is closely related to the catastrophic rupture strain and the rupture strain can be calculated in accord with the local-mean-field model satisfactorily. This provides a possible clue to the forecast of catastrophic rupture.
\end{abstract}

(C) 2007 Elsevier Ltd. All rights reserved.

Keywords: Localization; Scale of localized zone; Catastrophic rupture

\section{Introduction}

The catastrophic rupture of heterogeneous brittle media, like rocks and the earth's crust, presents complexity [1-9], especially exhibits sample-specific-

\footnotetext{
* Corresponding author. Address: State Key Laboratory of Nonlinear Mechanics, Institute of Mechanics, Chinese Academy of Sciences, Beijing 100080, China. Tel.: +86 10 62548133; fax: +861062579511.

E-mail address: hsw@lnm.imech.ac.cn (S.W. Hao).
}

ity [7-9]. It implies a great diversity of rupture thresholds for samples with identical initial macroscopic properties. This results in a tremendous difficulty in the prediction of catastrophic rupture. A possible strategy to deal with the problem is to search for some common, but specific precursors of catastrophe.

Numerous observations in laboratory compression tests on rocks and concrete revealed that the deformation becomes localized somewhere in a specimen ahead of the peak load [10-13]. On the other hand, it is well known that as soon as the 
elastic energy stored in the testing system, like the machine, becomes greater than the fracture energy of the specimen, the catastrophic rupture will occur $[12,14,15]$. However, it was observed that even for the so called stiff testing machine, catastrophic rupture still can happen [10-13]. It is supposed that this should be attributed to the localization of damage and deformation of specimen. But, the problem how the localization is related to the every value of catastrophic rupture strain remains open till now.

The strain localization has been analyzed as a bifurcation in the constitutive description of homogeneous deformation $[16,17]$. On the other hand, it was found recently that for heterogeneous media macroscopically localized damage may serve as an early precursor to rupture [18-20]. However, these previous works precludes post-localization analysis, hence unable to provide a definite relation between localization and rupture. In fact, no matter how thin it may be, a localized zone (or fracture zone) has its own width $[21,22]$. Therefore, to unveil the relationship between the localization and rupture is really a challenge and opportunity.

The objective of the present work is to elaborate the dependence of catastrophic transition of specimen on localized zone. Firstly, the results are outlined based on global mean-field approximation [23-25] and the driven nonlinear threshold model [26]. Then, an instrumented observation of spatiotemporal evolution of surface strain and catastrophic rupture of rock specimens under uniaxial compression is reported. As anticipated, the results based on global mean-field model deviate far from the observed values. This means that the global mean-field approximation is insufficient to represent rupture of a specimen because localization develops severely ahead of rupture. Therefore, our interests were focused on the formation of localization and the determination of the localized zone width. Actually, the evolution of surface strain pattern reflects the damage evolution of the sample and the width of damage localization zone is one of important parameters to predict the occurrence of rupture. Then, the analysis based on localmean-field approximation, i.e., applying mean-field approximation to the localized zone and its surrounding area separately, is introduced. According to the localized zone scale, the rupture strain in experiment can be calculated according to the analysis and the calculated and measured strains at rupture agree very well with each other.

\section{Rupture prediction based on global mean-field approximation}

In order to formulate the catastrophic rupture in heterogeneous media easily, the analysis is started with global mean-field approximation [23-25], To analyze the effects of mesoscopic heterogeneity, the Weibull probability distribution function [27]

$h\left(\sigma_{\mathrm{c}}\right)=\frac{m}{\eta}\left(\frac{\sigma_{\mathrm{c}}}{\eta}\right)^{m-1} \exp \left(-\left(\frac{\sigma_{\mathrm{c}}}{\eta}\right)^{m}\right)$

is adopted to characterize the strength distribution of mesoscopic elements in this model, where $\sigma_{\mathrm{c}}$ is the strength of mesoscopic elements, $\eta$ is proportional to mean strength, and $m$ is the shape parameter (Weibull modulus) characterizing the degree of heterogeneity. For an elastic mesoscopic element, the strain threshold is

$\varepsilon_{\mathrm{c}}=\frac{\sigma_{\mathrm{c}}}{E_{0}}$,

where $E_{0}$ is the elastic modulus of pristine state. So the expression (1) can be rewritten as

$h\left(\varepsilon_{\mathrm{c}}\right)=\frac{m}{\eta}\left(\frac{E_{0} \varepsilon_{\mathrm{c}}}{\eta}\right)^{m-1} \exp \left(-\left(\frac{E_{0} \varepsilon_{\mathrm{c}}}{\eta}\right)^{m}\right)$.

In light of the driven nonlinear threshold model [26], once an element reaches its stress threshold $\sigma_{\mathrm{c}}$ (strain $\varepsilon_{\mathrm{c}}$ ), it is assumed to be damaged, Accordingly, the continuum damage $D$ can be defined and expressed as

$D(\varepsilon)=\int_{0}^{\varepsilon} h\left(\varepsilon_{\mathrm{c}}\right) \mathrm{d} \varepsilon_{\mathrm{c}}=1-\exp \left[-\left(\frac{E_{0} \varepsilon}{\eta}\right)^{m}\right]$

where $\varepsilon$ denotes the nominal strain of the specimen. Here, global mean-field approximation is adopted, i.e., the fields of stress, strain and damage are assumed to be uniform, approximately. Then, according to continuum damage mechanics, the relation between stress and strain is

$\sigma=E_{0}(1-D(\varepsilon)) \varepsilon$.

The combination of Eqs. (4) and (5) gives the stressstrain relation,

$\sigma\left(E_{0} \varepsilon / \eta\right)=E_{0} \varepsilon \mathrm{e}^{\left(-\left(E_{0} \varepsilon / \eta\right)^{m}\right)}$.

The catastrophic rupture occurs when the slope of load-displacement curve of a specimen is equal to the unloading stiffness $-k_{\text {machine }}$ of test machine. So, if the fields of stress, strain and damage keep 
uniform until catastrophic rupture, the catastrophe condition can be expressed as $[12,14,15]$

$\frac{E_{0} A}{l}(1-D(\varepsilon)-\varepsilon h(\varepsilon))=-k_{\text {machine }}$.

where $A$ and $l$ are the cross area and the height of a specimen respectively. The left side of Eq. (7) is the slope of load-displacement curve of a specimen.

Once the probability distribution function of mesoscopic strength $h\left(\varepsilon_{c}\right)$ can be determined from experimental data, one can calculate the rupture strain $\varepsilon_{F, G}$ according to Eq. (7), where subscripts $F$ and $G$ denote rupture and global mean-field approximation, respectively.

In the following it will be described that how to experimentally determine the parameters $m, \eta$ and $E_{0}$ involved in the model [28]. Firstly, the slope of the experimental $\sigma-\varepsilon$ curve can be calculated (Fig. 1). Generally, the early increase in the measured $\mathrm{d} \sigma / \mathrm{d} \varepsilon$ is due to the elastic closing of cracks $[16,29]$ and has little influence on the following analysis. So, the point $Q$ corresponding to the maximum slope of the $\sigma-\varepsilon$ curve, is chosen as the starting point where the above mentioned model can be used, and an assumption is adopted that $(\mathrm{d} \sigma / \mathrm{d} \varepsilon)_{Q}$ is approximately equal to $E_{0}$. Then, it is supposed that the part of $\sigma-\varepsilon$ curve lower than point $Q$ is linear elastic with $E_{0}$ (a straight line) and shift the combined $\sigma-\varepsilon$ curve, i.e. the measured part beyond point $Q$ and the elastic part with $E_{0}$, horizontally till the origin, (see Fig. 1). Therefore, a processed integrated experimental nominal stress-strain curve (the dash line in Fig. 1) is obtained.

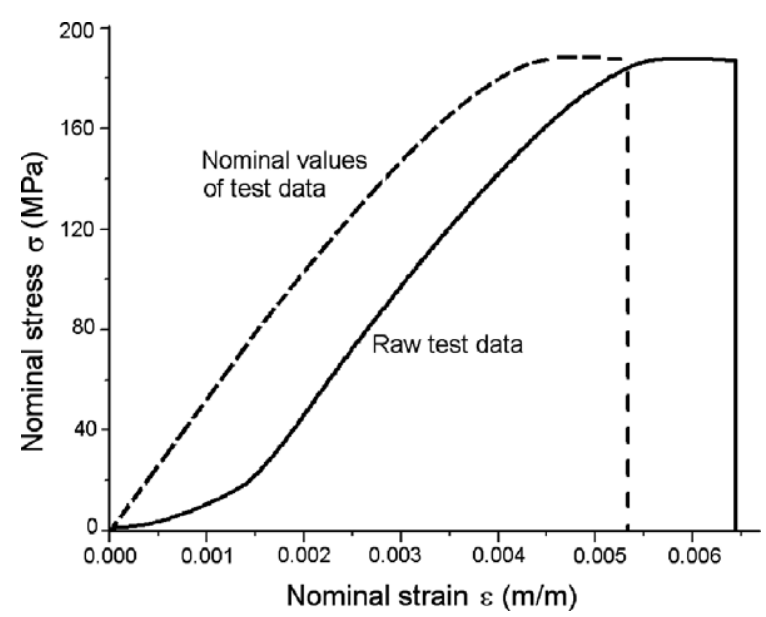

Fig. 1. Stress-strain curve for nominal test data raw test data.
Now, the other two parameters $m$ and $\eta$ can be obtained, by fitting the processed stress-strain curve to Eq. (5) and minimizing the following function,

$f\left(m, \eta, E_{0}\right)=\sum_{i}\left(\sigma_{i}\left(E_{0} \varepsilon_{t} / \eta\right)-\left(\sigma_{t} / \eta\right)_{i}\right)^{2}$,

where $\varepsilon_{t}$ and $\sigma_{t}$ are the processed experimental nominal stress and strain respectively, and $\sigma_{i}$ is the normalized stress of the model (Eq. (6)). The three fitting parameters of 6 specimens are shown in Table 1 respectively. With these parameters, a theoretical nominal stress-strain curve can be obtained in accord with Eq. (6). The agreement between the calculated and experimental nominal stress-strain curves appear quite good prior to the peak stress (see Fig. 2). Meanwhile, the calculated damage fraction at point $Q$ is much less than that at peak stress, e.g., for specimen 1 , the former is 0.004 and the later is 0.216 . So, the effects of damage before point $Q$ on the fitting parameters can be ignored.

The catastrophe transition of a specimen is due to the release of elastic energy stored in the testing system, like the testing machine [12,14,15] In our experiments, the stiffness $k_{\text {machine }}$ of the loading (including columns, hydraulics and platens) shows slight variation in the test as shown in Table 1.

Now, the rupture strain $\varepsilon_{\mathrm{F}, \mathrm{G}}$ of specimen can be calculated based on the global mean-field model and the stiffness $k_{\text {machine }}$ of testing machine, by means of Eq. (7). The calculated rupture strains $\varepsilon_{\mathrm{F}, \mathrm{G}}$ and their comparisons to the measured rupture strains $\varepsilon_{\mathrm{F}, \mathrm{m}}$ are shown in Table 1. Compared to the measured values, the mean relative deviation of calculated rupture strains in 6 tests is about $17 \%$. Clearly, the deviation is unacceptable in engineering. In particular, all the 6 calculated rupture strains are greater than those of measured values, hence the rupture forecast based on the global mean-field approximation seems to be too rough, even dangerous.

In fact, our experimental observations have demonstrated clear deformation localization ahead of rupture. Hence though the principle of rupture occurrence owing to the release of elastic energy stored in the testing system may remain valid, the global mean-field approximation fails to the rupture discussion. Therefore, it is necessary to pay special attention on damage and deformation localization experimentally and theoretically. In the following sections, some detailed experimental observations of deformation localization and the corresponding 
Table 1

Model parameters and the calculated values of rupture strain

\begin{tabular}{|c|c|c|c|c|c|c|}
\hline Specimens & 1 & 2 & 3 & 4 & 5 & 6 \\
\hline Model parameters & $\begin{array}{l}E_{0}=52.5 \mathrm{GPa} \\
\eta=459 \mathrm{MPa} \\
m=3.9\end{array}$ & $\begin{array}{l}E_{0}=52.5 \mathrm{GPa} \\
\eta=425 \mathrm{MPa} \\
m=2.7\end{array}$ & $\begin{array}{l}E_{0}=59.5 \mathrm{GPa} \\
\eta=412 \mathrm{MPa} \\
m=3.0\end{array}$ & $\begin{array}{l}E_{0}=90.5 \mathrm{GPa} \\
\eta=490 \mathrm{MPa} \\
m=2.6\end{array}$ & $\begin{array}{l}E_{0}=44.5 \mathrm{GPa} \\
\eta=356 \mathrm{MPa} \\
m=5.5\end{array}$ & $\begin{array}{l}E_{0}=78.5 \mathrm{GPa} \\
\eta=517 \mathrm{MPa} \\
m=3.3\end{array}$ \\
\hline Machine stiffness $(\mathrm{kN} / \mathrm{mm})$ & $k_{\mathrm{m}}=214.835$ & $k_{\mathrm{m}}=210.189$ & $k_{\mathrm{m}}=204.289$ & $k_{\mathrm{m}}=212.606$ & $k_{\mathrm{m}}=245.064$ & $k_{\mathrm{m}}=196.006$ \\
\hline Localized zone scale $\gamma(\mathrm{mm})$ & 6.7 & 7.9 & 7.9 & 6.9 & 7.1 & 6.3 \\
\hline $\begin{array}{l}\text { Measured values of rupture } \\
\text { strain } \varepsilon_{\mathrm{F}, \mathrm{m}}\end{array}$ & 0.00645 & 0.00655 & 0.00636 & 0.00515 & 0.00850 & 0.00634 \\
\hline $\begin{array}{l}\text { Predicted values based on } \\
\text { global mean-field theory } \varepsilon_{\mathrm{F}, \mathrm{G}}\end{array}$ & 0.00810 & 0.00839 & 0.00694 & 0.00661 & 0.00879 & 0.00682 \\
\hline Mean relative deviation & & & & $17 \%$ & & \\
\hline $\begin{array}{l}\text { Predicted values basing on } \\
\text { localized zone scale } \varepsilon_{\mathrm{F}, \mathrm{L}}\end{array}$ & 0.00674 & 0.00694 & 0.00611 & 0.00606 & 0.00816 & 0.00633 \\
\hline Mean relative deviation & & & & $6 \%$ & & \\
\hline
\end{tabular}

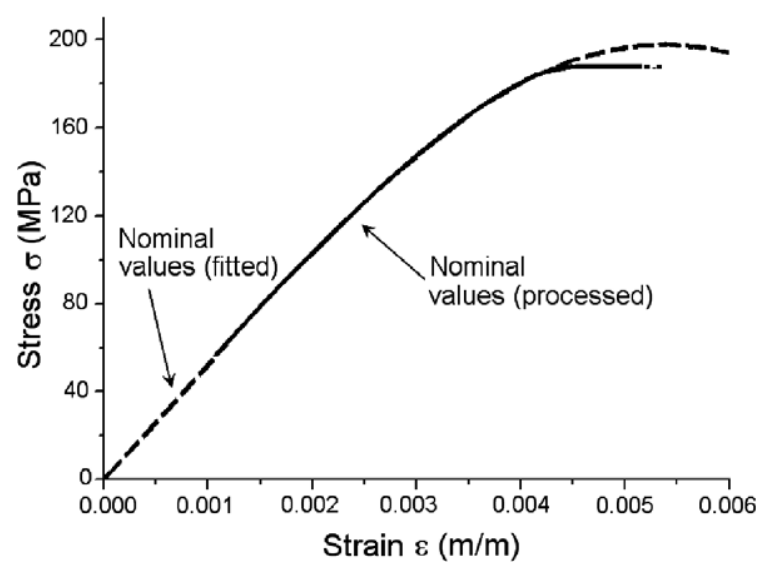

Fig. 2. Stress-strain curve for nominal test data: dotted portion fitted and solid portion processed.

local-mean-field approximation for the rupture forecast will be provided.

\section{Experimental method for surface strain-field measurement}

Digital speckle pattern metrology $[30,31]$ has been widely applied to the measurement of deformation of materials and structures. Also, digital speckle correlation method (DSCM) $[28,32]$ has been applied to measure deformation field of rock. In this paper, in order to explore the process from localization to eventual rupture, a similar digital speckle correlation method is adopted to examine the spatio-temporal pattern of the surface deformation fields of specimens.
The uniaxial compressive experiments were performed on granite. The testing machine is servocontrolled and the specimens with dimensions of $20 \times 16 \times 40 \mathrm{~mm}^{3}$ were loaded at a constant rate of $0.02 \mathrm{~mm} / \mathrm{min}$.

The digital speckle correlation method (DSCM) was coordinated with the loading system in order to detect the localization of deformation. The digital image, acquired by a charge-coupled-device (CCD) camera with a resolution of $768(\mathrm{H})$ and $576(\mathrm{~V})$, was immediately transmitted to image capturing board on a personal computer and recorded on a hard disk. After the experiment, the deformation fields of each test were calculated by the image analysis software developed by the Department of Modern Mechanics, The University of Science and Technology of China (USTC). The photographic resolution is about $0.05 \mathrm{~mm} / \mathrm{pixel}$. Before testing, calibration was carried out. The size of an element used to calculate strain is taken to be 30 pixels. The measured fluctuation of strain is less than $200 \mu \varepsilon$, whilst the rupture strain is greater than $2000 \mu \varepsilon$.

\section{Experimental results for evolution and localization of surface strain}

In this section, as an example, the data of specimen 1 (Figs. 4-8) will be analyzed. In all figures of this paper, the axis $x$ is set as vertical to the eventual rupture surface and its origin $(x=0)$ is just at the rupture surface (Fig. 3). In addition, time $t=0$ was set at the occurrence of the eventual rupture, so the negative values, like $t=-27 \mathrm{~s}$ (Fig. 4), means the time lag ahead of the rupture. As shown in 


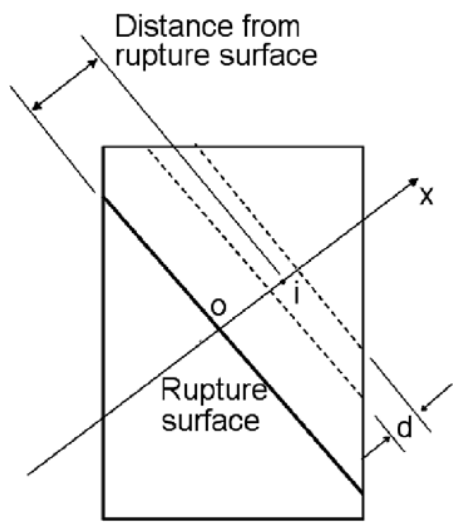

Fig. 3. Schematic of reference axis.

Fig. 4, the first principal strain $\varepsilon_{i}=\varepsilon\left(x_{i}\right)$ is the mean strain of a slice ( $2 \mathrm{~mm}$ in thickness) parallel to rupture surface at distance $x_{i}$. And the distance $x$ between the middle of this slice and the eventual rupture surface is named as the distance from rupture surface. Strain fluctuation is defined as $\varepsilon_{i}-\langle\varepsilon\rangle$, where $\langle\varepsilon\rangle$ represents the average strain of the whole specimen at the same time $(\langle\varepsilon\rangle=$ $\left.\sum_{i}^{I \text { (wholespecimen })} \varepsilon_{i} / I\right)$. Also, the derivative $\mathrm{d}\left(\varepsilon_{i}-\langle\varepsilon\rangle\right) /$ $\mathrm{d} t$ denotes the rate of strain fluctuation.

Figs. 4 and 5 show the distribution of $\varepsilon_{i}-\langle\varepsilon\rangle$ and $\mathrm{d}\left(\varepsilon_{i}-\langle\varepsilon\rangle\right) / \mathrm{d} t$ on the whole surface (Fig. 3) of specimen 1 at various times respectively. The fluctuations of strain are random at early stage $(t=-27 \mathrm{~s}$ ). Prior to rupture (from $t=-1.2 \mathrm{~s}$ to $t=0 \mathrm{~s}$ ), significant strain localization develops adjacent to the plane $x=0$.

A spatio-temporal pattern of the strain fluctuation of specimen 1 is shown in Fig. 6. It is found that there are two phases in the evolution of strain fields.

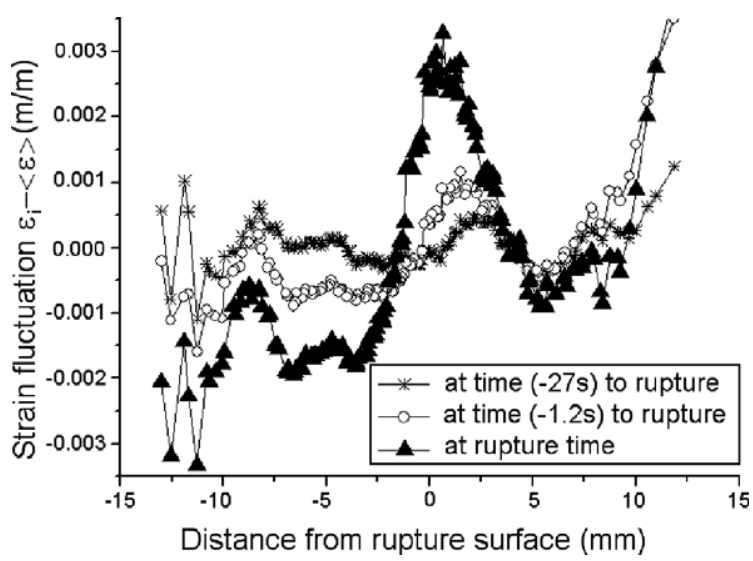

Fig. 4. Distribution of strain fluctuation at various time.

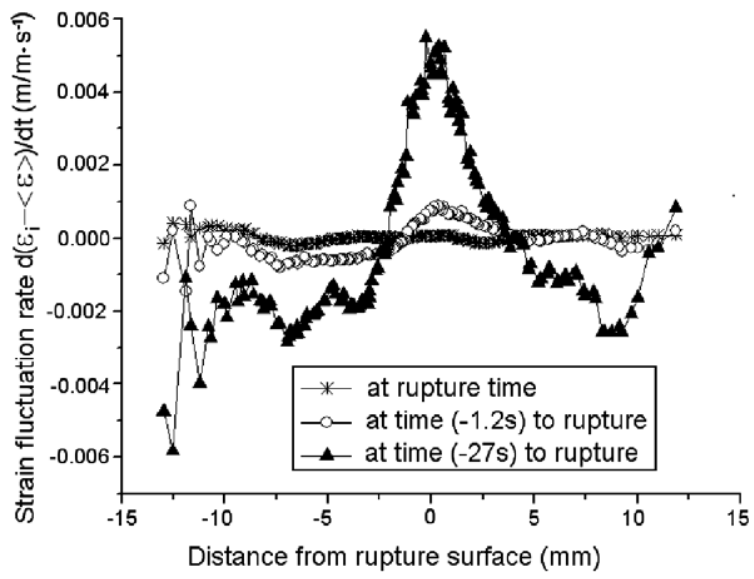

Fig. 5. Distribution of strain fluctuation rate at various time.

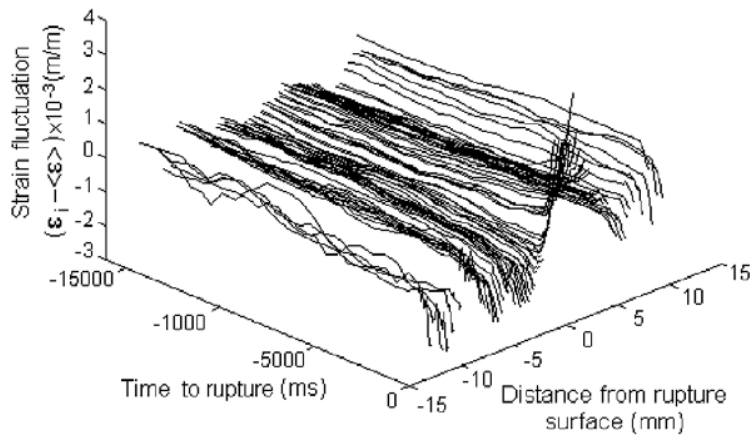

Fig. 6. Spatio-temporal pattern of strain fluctuation.

The duration of phase I is much longer than that of phase II. In phase I, the strain is nearly homogeneous with weak fluctuations, and the strain pattern evolves slowly. Afterwards, the nucleation and accelerating development of localization appear. This is phase II, which eventually leads to a significantly localized zone, namely the rupture surface.

In order to depict the accelerating evolution in phase II, the spatio-temporal pattern of strain fluctuation rate is shown in Fig. 7. From this figure, it is clear that the strain fluctuation rate in phase II increases rapidly in the localized zone with high strain.

The formation of localized zone can be attributed to the connection of spots with unstable development of local strain. In order to identify the unstable spots, the conditions

$\frac{\mathrm{d}\left(\varepsilon_{i}-\langle\varepsilon\rangle\right)}{\mathrm{d} t}>0$ and $\left(\varepsilon_{i}-\langle\varepsilon\rangle\right)>0$ 


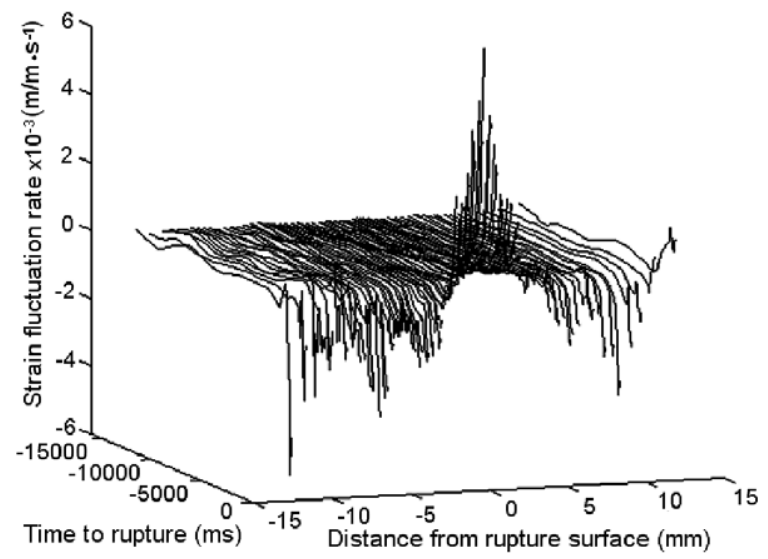

Fig. 7. Spatio-temporal pattern of strain fluctuation rate.

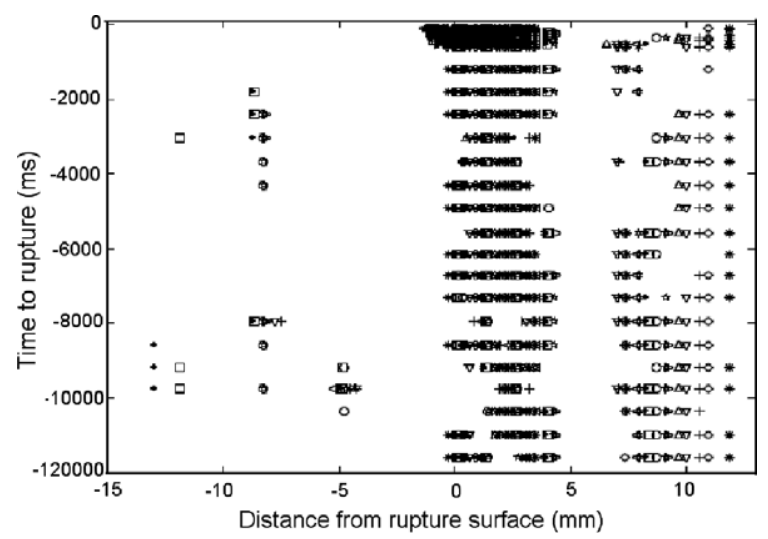

Fig. 8. Spatio-temporal pattern of unstable points on specimen. The marks represent satisfaction of condition (9).

were used as the unstable development of local strain. Fig. 8 shows a spatio-temporal pattern of the unstable points on the whole specimen. The marks in Fig. 8 represent the Spatio-temporal state at which $\varepsilon_{i}$ satisfies both $\left(\varepsilon_{i}-\langle\varepsilon\rangle\right)>0$ and $\frac{\mathrm{d}\left(\varepsilon_{i}-\langle\varepsilon\rangle\right)}{\mathrm{d} t}>0$. It can be observed that some unstable points on the specimen may disappear outside localized zone, whilst the others form a persistently localized zone, as the enlarged figure (Fig. 9) shows.

Hence, the localized zone at time $t$ can be defined to be the region, where both $\left(\varepsilon_{i}-\langle\varepsilon\rangle\right)>0$ and $\frac{\mathrm{d}\left(\varepsilon_{i}-\langle\varepsilon\rangle\right)}{\mathrm{d} t}>0$ are satisfied afterwards. Then, the width of localized zone can be determined accordingly. Fig. 9 shows the process that the unstable points in the localized zone become persistent when the unstable points outside this zone become disappear. The solid squares in Fig. 9 represent the spots, where the above-mentioned inequalities are satisfied

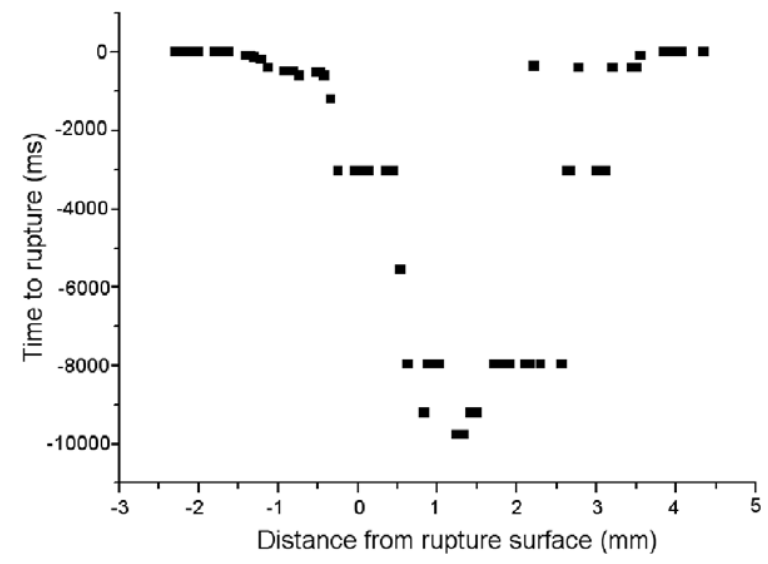

Fig. 9. Time-dependent range of the localized zone. The solid square represents satisfaction of condition (9) afterwards.

afterwards. Therefore, the width of eventual localized zone can be estimated to be $6.7 \mathrm{~mm}$, approximately, nearly one sixth of the specimen dimension. In addition, the eventual rupture surface forms within the localized zone.

\section{Local-mean-field approximation based on damage localization}

When localization of deformation and/or damage occurs, a specimen can be depicted as two parts, see Fig. 10, i.e. a localized zone with going on deformation and/or damage and a non-localized zone with potential release of elastic energy. As before the testing machine remains the other elastic part with stiffness $k_{\text {machine. }}$ At early stage, the same damage develops in the two parts. But after localization, the damage or deformation concentrates in the localized part. When the release of the elastic energy

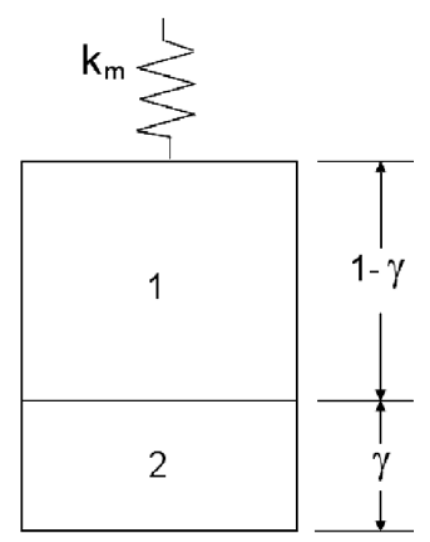

Fig. 10. Model consisting of localized and non-localized zones. 
stored in the machine as well as in the non-localized part become greater than the fracture energy of the localized part, a catastrophic rupture will appear.

In the following, a relation between the catastrophic rupture and the localized zone scale will be derived, based on a driven nonlinear threshold model and local-mean-field approximation. The elastic modulus of pristine specimen is assumed to be homogeneous and is made as unit. It is also assumed that both the height of specimen $l$ and the area of specimen's cross section $A$ are unit. Based on local-mean-field approximation, the damage fraction of part 1 and part 2 can be expressed as

$D_{1}=\int_{0}^{\varepsilon_{1}} h\left(\varepsilon_{\mathrm{c}}\right) \mathrm{d} \varepsilon_{\mathrm{c}}, \quad D_{2}=\int_{0}^{\varepsilon_{2}} h\left(\varepsilon_{\mathrm{c}}\right) \mathrm{d} \varepsilon_{c}$,

where $h\left(\varepsilon_{\mathrm{c}}\right)$ is initial probability distribution functions of breaking threshold $\varepsilon_{\mathrm{c}}$ of mesoscopic elements for part 1 and part 2. $h\left(\varepsilon_{\mathrm{c}}\right)$ is adopted as Weibull distribution functions provided $\eta$ is made as unit, i.e:

$h\left(\varepsilon_{\mathrm{c}}\right)=m \varepsilon_{\mathrm{c}}^{m-1} \mathrm{e}^{-\varepsilon_{\mathrm{c}}^{m}}$,

and Eq. (10) can be rewritten as

$$
\left.\begin{array}{l}
D_{1}=\int_{0}^{\varepsilon_{1}} h\left(\varepsilon_{\mathrm{c}}\right) \mathrm{d} \varepsilon_{\mathrm{c}}=1-\mathrm{e}^{-\varepsilon_{1}^{m}} \\
D_{2}=\int_{0}^{\varepsilon_{2}} h\left(\varepsilon_{\mathrm{c}}\right) \mathrm{d} \varepsilon_{\mathrm{c}}=1-\mathrm{e}^{-\varepsilon_{2}^{m}}
\end{array}\right\},
$$

The strain of a specimen can be expressed with the strain of these two parts as

$\varepsilon=\varepsilon_{1}(1-\gamma)+\varepsilon_{2} \gamma$,

where $\gamma=\frac{l_{2}}{l}=1-\frac{l_{1}}{l}$ is the relative scale of localized zone, $l_{1}$ and $l_{2}$ are the initial heights of part 1 and part 2 , respectively, and $l=l_{1}+l_{2}$. Here, part 2 corresponds to the localized zone of damage.

The normalized constitutive relation and the mechanical equilibrium condition can be expressed as

$\sigma=\sigma_{1}=\left(1-D_{1}\right) \varepsilon_{1}=\sigma_{2}=\left(1-D_{2}\right) \varepsilon_{2}$.

At the early stage of loading the specimen is weakly damaged and the strain field keeps uniform $\varepsilon_{1} \simeq \varepsilon_{2}$. The localization point, denoted by $\varepsilon=\varepsilon_{\mathrm{L}}$, is defined that the damage becomes mainly concentrated in one part, i.e., part 2, and no further damage will occur in part 1 . Thus, the damage fraction of part 1 is assumed to be a constant $D_{1}\left(\varepsilon_{\mathrm{L}}\right)=\int_{0}^{\varepsilon_{\mathrm{L}}} h\left(\varepsilon_{\mathrm{c}}\right) \mathrm{d} \varepsilon_{\mathrm{c}}$ (see Eq. (10) after the localization point.

The catastrophic rupture will appear at the higher damaged part, i.e., part 2. Then the rupture condition is that the slope of force-displacement curve of the part $2 \mathrm{~d}\left[\left(1-D_{2}\right) \varepsilon_{2}\right] /\left(\gamma \mathrm{d} \varepsilon_{2}\right)$ is equal to a negative value $-1 /\left((1-\gamma) /\left(1-D_{1}\right)+1 / k_{\mathrm{m}}\right)$, the stiffness of the combination of testing machine and part 1, i.e.

$\frac{\mathrm{d}\left[\left(1-D_{2}\left(\varepsilon_{2}\right)\right) \varepsilon_{2}\right]}{\gamma \mathrm{d} \varepsilon_{2}}=-\frac{1}{(1-\gamma) /\left(1-D_{1}\left(\varepsilon_{\mathrm{L}}\right)\right)+1 / k_{\text {machine }}}$,

where the damage fractions $D_{1}$ and $D_{2}$ are determined by Eq. (10) respectively. Eq. (14) with Eq. (10) gives the rupture strain of part $2 \varepsilon_{\mathrm{F}, 2}$ as a function of $k_{\text {machine, }} \varepsilon_{\mathrm{L}}$ and $\gamma: \varepsilon_{\mathrm{F}, 2}=\varepsilon_{\mathrm{F}, 2}\left(k_{\text {machine }}, \varepsilon_{\mathrm{L}}, \gamma\right)$. From Eqs. (12) and (13), the corresponding nominal rupture strain $\varepsilon_{\mathrm{F}, \mathrm{L}}$ can be derived as

$$
\begin{aligned}
& \varepsilon_{\mathrm{F}, \mathrm{L}}\left(k_{\text {machine }}, \varepsilon_{\mathrm{L}}, \gamma\right) \\
& =\frac{\left(1-D_{2}\left(\varepsilon_{\mathrm{F}, 2}\left(k_{\text {machine }}, \varepsilon_{\mathrm{L}}, \gamma\right)\right)\right) \varepsilon_{\mathrm{F}, 2}\left(k_{\text {machine }}, \varepsilon_{\mathrm{L}}, \gamma\right)}{1-D_{1}\left(\varepsilon_{\mathrm{L}}\right)}(1-\gamma) \\
& \quad+\gamma \varepsilon_{\mathrm{F}, 2}\left(k_{\text {machine }}, \varepsilon_{\mathrm{L}}, \gamma\right),
\end{aligned}
$$

where subscript L denotes local-mean-field approximation. Clearly, the nominal rupture strain $\varepsilon_{\mathrm{F}, \mathrm{L}}$ is dependent on $\gamma$, the scale of localized zone. In addition, Eq. (15) together with Eqs. $\left(10^{\prime}\right),(11)$ and (14) can give the calculated rupture strain $\varepsilon_{\mathrm{F}, \mathrm{L}}$.

Now, a case with shape parameter $m=3$ is considered and its damage localization transition appears at the maximum load. Subsequently, loading and unloading appear in part 2 and part 1 separately. In this case, $\varepsilon_{\mathrm{L}}=\varepsilon_{\sigma_{\max }}$. The dependence of catastrophic rupture point on the scale of damage localization zone is shown in Fig. $11\left(\gamma\right.$ vs. $\left.\varepsilon_{2}\right)$ and Fig. $12\left(\varepsilon_{\mathrm{F}, \mathrm{L}}\right.$ vs. $\left.\gamma\right)$, where the normalized stiffness of testing machine is taken as $k_{\text {machine }}=1.433$. It is found that the nominal strain at catastrophic rupture $\varepsilon_{\mathrm{F}, \mathrm{L}}$ decreases with decreasing $\gamma$. In other words, the higher localization, i.e., the smaller scale of localized zone, the higher risk of catastrophic rupture.

It is noticeable that the damage localization generally appears before catastrophic rupture in the system initially described by uniform statistical distribution function with unique shape parameter $m$, owing to the deviations of damage field and stress field from the mean-field approximation. In fact, the damage localization transition and the scale of localized zone are related to mesoscopic details, i.e., the coupling between disordered mesoscopic heterogeneity and the microdamages will induce the redistribution of stress field. 


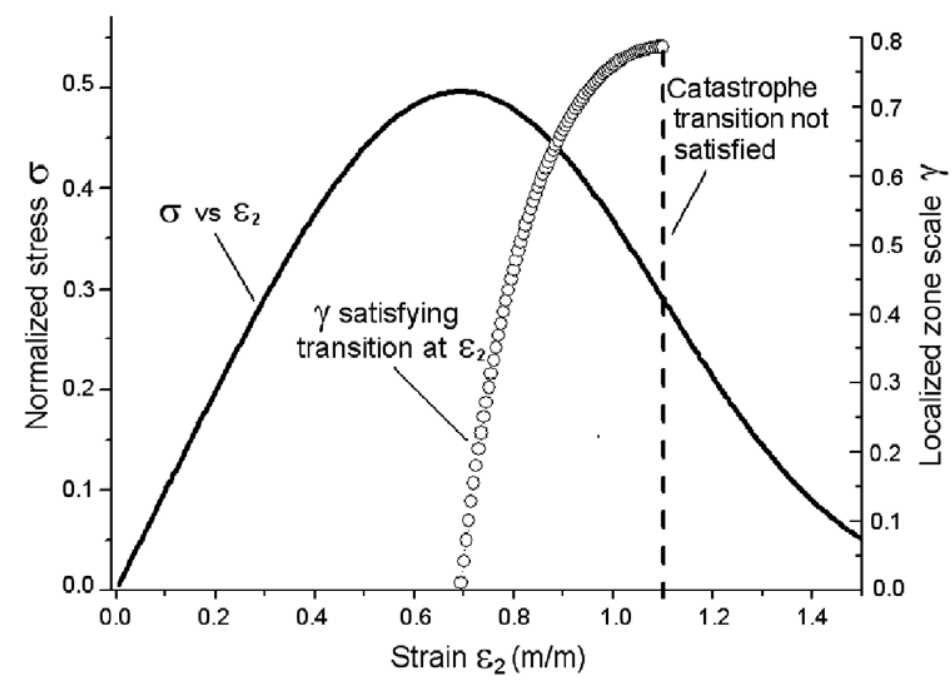

Fig. 11. Stress-strain relation of part 2 localized zone and localized zone scale $\gamma$.

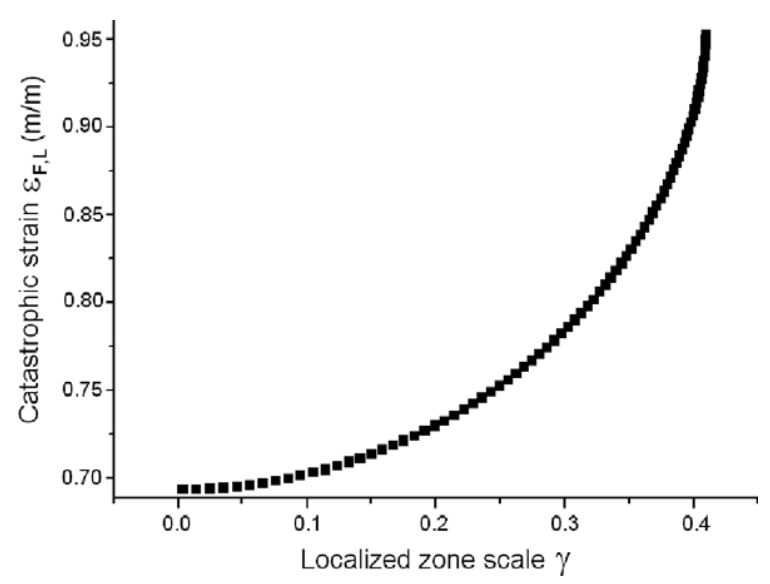

Fig. 12. Dependence of catastrophic strain $\varepsilon_{\mathrm{F}, \mathrm{L}}$ of specimen on localized zone scale $\gamma$.

\section{Rupture prediction based on localized scale}

Now, the rupture strain of specimen can be predicted by making use of Eqs. (13) and (14) based on the obtained parameters $\left(m, \eta, E_{0}\right.$ and $\left.k_{\text {machine }}\right)$ and the experimentally observed localized zone scale $\gamma$. The calculated strains $\varepsilon_{\mathrm{F}, \mathrm{L}}$ at rupture of 6 specimens are shown in Table 1 . These values are very closer to the observed ones. The mean relative deviation $6 \%$ of the results based on the local mean-field approximation is much less than that of global mean-field approximation (17\%). In addition, unlike the too great rupture strains obtained by the global mean-field model, a half of the calculated rupture strains based on the local mean-field approximation are less than the observed values, hence the local mean-field model is much more meaningful.

\section{Discussions and conclusions}

The experimental observations demonstrate that there are two phases in the spatio-temporal surface strain pattern of granite under compression. The later phase II is characterized by an accelerating localization and is closely related to catastrophic rupture. The accelerating evolution of localized strain in phase II implies a sensitive response of the specimen to external loading prior to the eventual rupture. Also, the eventual rupture surface is located in the localized zone with high strain. Hence, the investigation of the relation between strain localization and catastrophic rupture is crucial to the search for precursors of rupture.

In comparison with the experimental measurements, the global mean-field approximation always leads to much later rupture occurrence, hence, it seems to be to rough and even dangerous in rupture forecast. Obviously, this is because the global meanfield approximation neglects the appearance of localized zone ahead of rupture. However, together with experimental results before the peak stress, the approximation can provide a necessary estimation of the heterogeneous parameters of specimen. These parameters, like the shape factor of Weibull distribution, are significant in catastrophic rupture.

With these obtained parameters and based on local mean-field approximation, which takes the 
appearance of localization into account, it is found that the strain of catastrophic rupture can be calculated properly, provided the localized zone scale is available experimentally. The calculated rupture strains are in good agreement with the experimental results, with mean deviation $6 \%$. So, the local meanfield model is a very promising approach to the forecast of catastrophic rupture.

\section{Acknowledgements}

This work is supported by the NSFC (Nos. 10302029, 10472118, 10372012 and 10232050). The authors thank Dr. Hong Miao for his kind help in measurements.

\section{References}

[1] P. Meakin, Models for material failure and deformation, Science 252 (1991) 226-234.

[2] D.R. Curran, L. Seaman, D.A. Shockey, Dynamic failure of solids, Physics Reports 147 (1997) 253-388.

[3] W.A. Curtin, Toughening in disordered brittle materials, Physical Review B 55 (1997) 11270-11276.

[4] Y. Ben-zion, C.G. Sammis, Characterization of fault zones, Pure and Applied Geophysics 160 (2003) 677-715.

[5] S.C. Jaume, L.R. Sykes, Evolving towards a critical point: a review of accelerating seismic moment/energy release prior to large and great earthquakes, Pure and Applied Geophysics 155 (1999) 279-305.

[6] J.B. Rundle, W. Klein, K.F. Tiampo, S. Gross, Linear pattern dynamics in nonlinear threshold systems, Physical Review E 61 (2000) 2418-2432.

[7] M.F. Xia, Z.Q. Song, J.B. Xu, K.H. Zhao, Y.L. Bai, Sample - specific behavior in failure models of disordered media, Communications in Theoretical Physics 25 (1) (1996) 49-54.

[8] M.F. Xia, F.J. Ke, J. Bai, Y.L. Bai, Threshold diversity and trans-scales sensitivity in a finite nonlinear evolution model of materials failure, Physics Letters A 236 (1997) 60-64.

[9] Y.L. Bai, H.Y. Wang, M.F. Xia, F.J. Ke, Statistical mesomechanics of solid, linking coupled multiple space and time scales, Applied Mechanics Reviews 58 (2005) 372-388.

[10] W.R. Wawersik, C.A. Fairhurst, A study of brittle rock fracture in laboratory compression experiments, International Journal of Rock Mechanics and Mining Sciences 7 (1970) 561-575.

[11] T.F. Wong, Micromechanics of faulting in westerly granite, International Journal of Rock Mechanics and Mining Science and Geomechanics Abstracts 19 (1982) 49-64.

[12] J.F. Labuz, L. Biolzi, Class I vs. class II stability: a demonstration of size effect, International Journal of Rock Mechanics and Mining Science and Geomechanics Abstracts $28(2 / 3)(1991)$ 199-205.

[13] S. Sture, H.Y. Ko, Strain-softening of brittle geologic materials, International Journal for Numerical and Analytical Methods in Geomechanics 2 (1978) 237-253.
[14] M.D.G. Salamon, Stability, instability and design of pillar workings, International Journal of Rock Mechanics and Mining Sciences 7 (6) (1970) 613-631.

[15] J.A. Hudson, S.L. Crouch, C. Fairhurst, Soft, stiff and servocontrolled testing machine: a review with reference to rock failure, Engineering Geology 6 (1972) 155-189.

[16] J.W. Rudnicki, J.R. Rice, Conditions for localization of deformation in pressure-sensition dilatant materials. 23 (1975) 371-394.

[17] I. Vardoulakis, Shear band inclination and shear modulus of sand in biaxial tests, International Journal for Numerical and Analytical Methods in Geomechanics 4 (1980) 103-119.

[18] Y.L. Bai, M.F. Xia, F.J. Ke, H.L. Li, Damage field equation and criterion for damage localization, in: R. Wang (Ed.), Rheology of Bodies with Defects, Proceedings of IUTAM Symposium, Kluwer Academic Publishers, Dordrecht, 1998, pp. 55-66.

[19] H.L. Li, Y.L. Bai, M.F. Xia, F.J. Ke1, X.C. Yin, Damage localization as a possible precursor of earthquake rupture, Pure and Applied Geophysics 157 (2000) 1929-1943.

[20] H.Y. Wang, Y.L. Bai, M.F. Xia, F.J. Ke, Spallation analysis with a closed trans-scale formulation of damage evolution, Acta Mechanica Sinica (4) (2004) 400-407.

[21] L. Anand, W.A. Spitzig, Initiation of localized shear bands in plane strain, Journal of the Mechanics and Physics of Solids (28) (1980) 113-128.

[22] M. Ohnaka, A constitutive scaling law and a unified comprehension for frictional slip failure, shear fracture of intact rock, and earthquake rupture, Journal of Geophysical Research 108 (B2) (2003) 2080, doi:10.1029/2000JB000123.

[23] L.M. Kachanov, On the time to Failure Under Creep Conditions, Lzv. Akad. Nauk SSSR, Otd. Tekh. Nauk. Metall. Topl. 8 (1958) 26-61.

[24] J. Lemaitre, J.-L. Chaboche, Mecanique des Materiaux Solides, Dunod, Paris, 1985.

[25] D. Krajcinovic, Damage Mechanics, North-holland, Amsterdam, The Netherlands, 1996.

[26] X.H. Zhang, X.H. Xu, H.Y. Wang, M.F. Xia, F.J. Ke, Y.L. Bai, Critical sensitivity in driven nonlinear threshold systems, Pure and Applied Geophysics 161 (2004) 1931-1944.

[27] W. Weibull, A statistical distribution function of wide applicability, Journal of Applied Mechanics 18 (1951) 293297.

[28] X.H. Xu, S.P. Ma, M.F. Xia, F.J. Ke, Y.L. Bai, Damage evolution and damage localization of rock, Theoretical and Applied Fracture Mechanics 42 (2004) 131-138.

[29] J.C. Jaeger, N.G.W. Cook, Fundamentals of Rock Mechanics, third ed., Chapman and Hall, London, 1979.

[30] Z. Jiang, Q. Zhang, H. Jiang, Z. Chen, X. Wu, Spatial characteristics of the Portevin-Le Chatelier deformation bands in Al-4 at.\% $\%$ polycrystals, Materials Science and Engineering A 403 (2005) 154-164.

[31] J.N. Perie, S. Calloch, C. Cluzel, F. Hild, Analysis of a multiaxial test on a $\mathrm{C} / \mathrm{C}$ composite by using digital image correlation and a damage model, Experimental Mechanics 42 (2002) 318-328.

[32] X.H. Xu, S.P. Ma, M.F. Xia, F.J. Ke, Y.L. Bai, Synchronous multi-scale observations on rock damage and rupture, Theoretical and Applied Fracture Mechanics 44 (2005) 146156. 